\title{
LA CYBERJUSTICE COMME RÉPONSE AUX BESOINS JURIDIQUES DES PERSONNES ITINÉRANTES: SON POTENTIEL ET SES EMBÛCHES
}

\author{
Suzanne Bouclin* \\ Marie-Andrée Denis-Boileau ${ }^{* *}$
}

Le présent texte cherche à déterminer si le développement de la cyberjustice améliorera ou compromettra l'accès à la justice pour les personnes en situation d'itinérance. Nous commencerons par une revue détaillée de la littérature actuelle sur la cyberjustice, pour ensuite présenter notre définition de la cyberjustice et de son rôle dans la promotion de l'accès à la justice. Dans une dernière section davantage exploratoire, nous élaborerons quelques propositions informelles de réponse à la question formulée précédemment - à savoir si la cyberjustice, dans ses formes actuelles, peut contribuer à répondre aux besoins juridiques des personnes en situation d'itinérance. Ces réponses seront évaluées dans une recherche ultérieure.

This aim of this paper is to determine whether the expansion of cyberjustice will improve or impede access to justice for individuals who are homeless. We begin with a detailed review of the current literature on cyberjustice, followed by our definition of cyberjustice and the role it plays in promoting access to justice. The last section, which is more exploratory in nature, offers a number of informal proposals for answering the question of whether cyberjustice, in its present forms, can help to meet the legal needs of homeless persons. Further research will be done in order to evaluate those answers.

\section{INTRODUCTION}

Le croisement entre le droit et la technologie ${ }^{1}$ attire, depuis maintenant plus d'une décennie, l'attention de la communauté juridique universitaire. Un vaste éventail de sujets découlant de ce croisement ont été

* $\quad$ Professeure adjointe, Faculté de droit, Université d’Ottawa. L’auteure principale exprime sa reconnaissance aux participants de la rencontre pré-ICIS francophone (Milan, 2013), au réviseur Philippe Ducharme, à Charlotte ChicoineWilson pour ses commentaires utiles et aux évaluateurs pour le temps qu'ils et elles ont consacré à la lecture de versions antérieures. Leurs commentaires éclairés et leurs suggestions sont fort appréciés. La réalisation de ce texte a été rendue possible grâce à l'appui financier du Conseil de recherche en sciences humaines du Canada. Les opinions exprimées n'engagent que les auteures.

** Candidate à la maîtrise en droit (LL.M.) à l’Université d’Ottawa et membre du Barreau du Québec depuis 2011.

1 Notre travail s'inscrit dans une perspective pluraliste du droit et de la technologie. Néanmoins, nous constatons que la majorité des textes qui interrogent le croisement de ces disciplines se réfèrent au droit officiel et formalisé (plutôt qu’à ses dimensions inférentielles, implicites et dynamiques) et à la technologie comme objet matériel (plutôt qu'artéfact social modifié par l'action humaine et modifiant celle-ci). Voir discussion pluraliste du droit : Martha Marie Kleinhans et Roderick A. Macdonald, «What is a Critical Legal Pluralism » (1998) 12 CJLS / RCDS 25; pour une œuvre qui rejoint les aspects sociaux de la technologie, voir W. J. Orlikowski, « The duality of technology: rethinking the concept of technology in organizations » (1992) 3:3 Organization science 398. 
étudiés, que ce soit la réglementation du cyberespace ${ }^{2}$, les conceptions émergentes de la propriété intellectuelle et du droit d'auteur ${ }^{3}$, la structure juridique encadrant la rencontre des technologies de l'information et de la communication (TIC) ainsi que du droit dans le contexte mondial actuel ${ }^{4}$, la vie privée et la sécurité ${ }^{5}$ ou la réglementation de l'identité ${ }^{6}$. D’autres auteurs se penchent plutôt sur les changements profonds opérés par les technologies de l'information et de la communication sur la distribution des services juridiques ${ }^{7}$ et sur le développement et la formation des connaissances juridiques ${ }^{8}$. Ces multiples recherches démontrent un intérêt grandissant pour la cyberjustice, un concept que nous définissons comme étant « l'intégration des TIC au monde judiciaire » 9 .

Il en résulte que l'étude des conséquences de l'intégration de toute forme de cyberjustice au monde judiciaire attire de plus en plus d'attention à l'échelle internationale ${ }^{10}$. Nous souhaitons apporter notre

2 Lawrence Lessig, « Code and Other Laws of CyberSpace » (1999), en ligne: Code is Law: <http://code-is-law.org> et Lawrence Lessig, « CodeV2 » (2006), en ligne: Code V2 <http://codev2.cc/>.

3 Carys J Craig, Joseph F Turcotte et Rosemary J Coombe, « What's Feminist About Open Access? A Relational Approach to Copyright in the Academy »(2001), en ligne : feminists@law

<http://journals.kent.ac.uk/index.php/feministsatlaw/article/view/7/54>; Ysolde Gendreau, An Emerging Intellectual Property Paradigm: Perspectives from Canada, Cheltenham, Edward Elgar, 2008; David Lametti, «The Form and Substance of Domain Name Arbitration », en ligne : (2002) 7:2 Lex Electronica < http://www.lexelectronica.org/articles/v7-2/lametti.htm >; Diane Rowland et Elizabeth Macdonald, Information Technology Law, Sydney, Cavendish, 2005; Teresa Scassa, « Extension of Intellectual Property Rights » dans David Vaver, Marcel Boyer et Michael Trebilcock, dir, Competition Policy and Intellectual Property, Toronto, Irwin Law, 2009, 17; A Strowel, Quand Google défie le droit, Bruxelles, Larcier, 2011.

4 Irene Maria Portela et Maria Manuela Cruz-Cunha, ICT Law, Protection and Access Rights: Global Approaches and Issues, New York, Hershey, 2010.

5 Vincent Gautrais, « Droit et sécurité : pas si sûr ! » (2007), en ligne : Gautrais.com <http://www.gautrais.com/IMG/pdf/PLAN_2007_Notarius.pdf>; Michael A. Geist et al, « Copyright \& Privacy Through the Technology Lens » (2005) 4:2 J Marshall Rev Int'l Prop L 242; Lesley Jacobs, Barbara Crow et Kim Sawchuk, « Privacy Rights Mobilization Among Marginal Groups in Canada: Fulfilling the Mandate of PIPEDA » (Mars 2011), en ligne : York Centre for Public Policy \& Law < http://ycppl.info.yorku.ca/files/2013/05/Privacy-RightsPIPEDA-paper.pdf>; Avner Levin, Patricia Sánchez Abril, « Two Notions of Privacy Online » (2009) 11 Vand J Ent \& Tech L 1001.

$6 \quad$ Bert-Jaap Koops et Ronald E. Leenes, « ID Theft, ID Fraud and/or ID-related Crime. Definitions Matter » (2006) 30:9 Datenschutz und Datensicherheit 553.

7 M. Ethan Katsh, Law in a Digital World, New York, Oxford University Press, 1995; Richard Susskind, The End of Lawyers? Rethinking the Nature of Legal Services, New York, Oxford University Press, 2010 [Susskind].

8 Daniel Poulin, «La démocratisation de l'accès au droit : nouveaux acteurs et nouvelles technologies » dans Claire Monville, dir, Variations sur le droit de la société de l'information, Bruxelles, Cahier du CRID, 2001, 27; Daniel Poulin, «Fifteen Years of Free Access to Law » dans Ginevra Peruginelli et Mario Ragona, dir, Law via the Internet Free Access, Quality of Information, Effectiveness of Rights, Florence, European Press Academic Publishing, $2008,15$.

9 Cette définition s'inspire d'autres travaux dans le domaine de la cyberjustice, voir par ex infra notes 28, $45,47$.

10 Voir par ex les travaux du Hague Institute for the Internationalization of Law, un institut de recherche ayant comme objectif l'internationalisation du droit dans un monde où les frontières nationales au sens traditionnel deviennent de moins en moins importantes pour certains citoyens, et où l'interconnectivité des sociétés, et donc également celle des différents systèmes de droit, est considérée comme un fait. Le cœur de leurs recherches porte sur les procédés de résolution des conflits pour lesquels l'innovation est essentielle: les TIC sont donc souvent à l'honneur dans leurs recherches. Voir Site internet du Hague Institute, en ligne: HiiL <http://www.hiil.org>. Dans la même lignée, la Commission des Nations Unies pour le droit commercial international (CNUDCI) souhaite moderniser et harmoniser les règles du commerce international. La CNUDCI travaille à établir des principes et des pratiques qui réduiront les divergences dans les domaines liés aux nouvelles pratiques commerciales telles que le commerce électronique : Site 
contribution en tentant de voir si la cyberjustice est un bon outil pour améliorer l'accès à la justice des personnes itinérantes $^{11}$. Présentement, au Québec et au Canada, de nombreuses personnes n’ont pas d'accès significatif à la justice formelle ${ }^{12}$. Les recherches indiquent que la plupart des Canadiens et Canadiennes à revenu moyen ne peuvent s'offrir les services d'un avocat ${ }^{13}$ et que les coûts élevés des services juridiques et l'inaccessibilité de l'aide juridique ${ }^{14}$ les contraignent à se représenter euxmêmes ${ }^{15}$. Malgré cette réalité troublante, il demeure que les Canadiens qui vivent dans la pauvreté, les travailleurs sous le seuil de la pauvreté et ceux à faible revenu sont toujours aux prises avec des problèmes juridiques non résolus ${ }^{16}$, ont des démêlés avec la justice ${ }^{17}$ ou font face à divers problèmes juridiques en matières civiles concernant la garde des enfants, les modalités du droit de visite, l'emploi et l'invalidité, et ce, plus fréquemment que les Canadiens à revenu plus élevé ${ }^{18}$. Toutefois, cette réalité

internet de la Commission des Nations Unies pour le droit commercial international, en ligne : CNUDCI $<$ http://www.uncitral.org/uncitral/fr/index.html>. CNUDCI, Guide de la CNUDCI: L'essentiel sur la Commission des Nations Unies pour le droit commercial international, Vienne, CNUDCI, 2013 à la p 14.

11 Cette population a fait l'objet de recherche insuffisante dans le domaine de l'accès à la justice. Dans son rapport sur les besoins juridiques des Ontariens, le « Projet sur les besoins juridiques civils de l'Ontario » a exclu les personnes itinérantes, citant comme raison que « les sans-abri ne pouvaient être sondés par téléphone de façon fiable », Comité directeur du Projet sur les besoins juridiques civils de l'Ontario, À l'écoute de l'Ontario : Rapport du Projet sur les besoins juridiques civils de l'Ontario, Toronto, Le Comité directeur du Projet sur les besoins juridiques, 2010 à la p 21.

12 Michael Trebilcock, Anthony Duggan et Lorne Sossin, « Introduction » dans Michael Trebilcock, Anthony Duggan et Lorne Sossin, dir, Middle Income Access To Justice, Toronto, University of Toronto Press, 20121 à la p 1.

13 Projet sur les besoins juridiques civils de l’Ontario, supra note 13 aux pp 35-36.

14 Les critères financiers pour l'admission sont si bas que les personnes ayant un salaire moyen font trop d'argent pour être admises à l'aide juridique, mais elles sont également incapables de payer les services d'un avocat (le tarif horaire moyen est présentement autour de 350 \$), voir Michael McKiernan, « Lawyers Integral in Making Justice Accessible: McLachlin » (21 février 2011), en ligne : Law Times online <http://www.lawtimesnews.com/201102218262/HeadlineNews/Lawyers-integral-in-making-justice-accessible-McLachlin>; Buckley, infra note 109 à la p 9; L'admissibilité financière ne tient pas compte du coût de la vie qui est de plus en plus élevé dans les centres urbains, voir Russell Engler, « Opportunities and Challenges: Non-Lawyer Forms of Assistance in Providing Access to Justice for Middle-Income Earners » dans Michael Trebilcock, Anthony Duggan et Lorne Sossin supra note 12 à la p 148; Au Québec, le seuil d'admissibilité à l'aide juridique est égal à 65 p. 100 du salaire minimum, voir Barreau du Québec, « Bilan de l'état de droit au Québec : Rapport du Barreau du Québec » (janvier 2012), en ligne : Barreau du Québec <http://www.barreau.qc.ca/pdf/publications/2012-bilan-etat-droit.pdf> à la p 37.

15 Julie Macfarlane, « The National Self-Represented Litigants Project: Identifying and Meeting the Needs of Self-Represented Litigants », mai 2013 [non publié], archivé en ligne :

$<$ http://www.representing-yourself.com/PDF/reportM15.pdf>] à la p 39; Buckley, infra note 109 aux pp 9-10.

16 Ab Currie, « A National Survey of the Civil Justice Problems of Low and Moderate Income Canadians: Incidence and Patterns » (2006) 13:3 Int’l J Legal Prof à la p 239.

17 Aide juridique Ontario a constaté une augmentation du nombre de personnes demandant l'aide juridique. Cette augmentation est due à une présence policière et des services de protection de l'enfance accrus dans les communautés à faible revenu, voir Buckley, infra note 23 à la p 51; voir aussi Buckley, infra note 109 à la p 8.

18 Sur une période de trois ans, près de la moitié des Canadiens à faible ou moyen revenu sondés par le ministère de la Justice a rencontré au moins un problème juridique significatif, voir Currie, supra note 16 à la p 239 (Ce sondage a été effectué auprès de personnes de plus de 18 ans ayant un revenu personnel de moins de 35000 \$ ou un revenu familial de moins de 50000 \$); Voir aussi R Roy McMurtry et Lorne Mitchell Sossin, Listening to Ontarians: Report of the Ontario Civil Legal Needs Project, Toronto: Ontario Civil Legal Needs Project Steering Committee, 2010 aux pp 34-35; Buckley, infra note 23 à la p 39. 
est amplifiée pour les personnes marginalisées historiquement ${ }^{19}$. C'est pourquoi nous avons choisi de nous concentrer sur la réalité d'un groupe marginalisé, savoir les personnes itinérantes. Celles-ci, selon certains auteurs, font moins confiance au système de justice que les personnes ayant un logement stable $^{20}$, comprennent moins bien le droit formel ${ }^{21}$ et sont moins bien outillées pour faire valoir leurs droits $^{22}$. Nous notons cependant que les propositions que nous formulerons relativement à ces personnes sont pertinentes pour plusieurs autres communautés marginalisées.

Dans un premier temps, nous expliquerons en détail ce qu'est la cyberjustice. Outre certaines exceptions pertinentes, cette discussion se limitera aux pays de common law et portera plus précisément sur le contexte canadien. Dans un deuxième temps, nous examinerons la relation entre les populations marginalisées, plus particulièrement les personnes itinérantes, et les TIC. Nous constaterons à cette étape que peu de recherches ont étudié les effets de la cyberjustice sur les populations vulnérables ou démunies dans leur rapport au système de justice ${ }^{23}$. En conséquence, nous nous questionnerons dans un troisième temps sur la pertinence de la cyberjustice comme l'a justice réponse aux besoins juridiques des personnes itinérantes. ${ }^{24}$

\section{QU'EST-CE-QUE LA CYBERJUSTICE?}

La cyberjustice se définit comme l'intégration des technologies de l'information et de la communication [TIC] au monde judiciaire ${ }^{25}$. Elle poursuit deux objectifs qui seront détaillés dans les prochains paragraphes : améliorer l’accessibilité à la justice pour tous, principalement en intégrant les

19 Currie, supra note 16 à la p 232. Voir aussi par ex Amber Kellen, Julie Freedman, Sylvia Novac, Linda Lapointe, Richard Maaranen et Angeline Wong, « Homeless and Jailed: Jailed and Homeless » (août 2010), en ligne : La Société John Howard du Canada <http://www.johnhoward.ca/document/JHS

Toronto\%20Report\%20Homeless\%20and\%20Jailed.pdf>.

20 Pierre Noreau, « La scolarité, la socialisation, et la conception du droit : un point de vue sociologique » (1997) 38:4 C de D 741 à la p 754; voir aussi Isabelle Raffestin, Une injustice programmée?: le point de vue des personnes itinérantes sur leur judiciarisation et leur incarcération, mémoire de maîtrise en service social, Université de Montréal, 2009 [non publié] à la p 113; Isabelle Raffestin, «L’avis des personnes itinérantes sur leur situation judiciaire », en ligne : (2010) $23: 119$ Porte ouverte <http://www.asrsq.ca/fr/pdf/po/por_1002.pdf>.

21 Eyrich-Garg, infra note 117 à la p 760.

22 Tel que l'expliquent Suzie Forrel et ses coauteurs, Suzie Forell, Emily McCarron et Louis Schetzer, No home, no justice? The legal needs of homeless people in NSW, Sydney, Law and Justice Foundation of New South Wales, 2005 à la p 115 (« [o]n a day to day basis, homeless people have many immediate needs: finding accommodation, getting food or money, caring for family. These needs tend to take precedence over their legal issues »).

23 Voir cependant Melina Buckley, Aide juridique - La voie du progrès : Recherche sur les besoins actuels et les approches novatrices (juin 2010), en ligne : Association du Barreau canadien

<http://www.cba.org/cba/Advocacy/PDF/CBA\%20Legal\%20Aid\%20Renewal\%20Paper.pdf> à la p 16 [Buckley 2010].

24 Nous utilisons le terme " itinérant » (en anglais « street involved people »), qui est utilisé dans les milieux militants, plutôt que les expressions plus communes comme sans-abri, personne de la rue, personne sans domicile fixe, ou. en anglais, « homeless ». Voir le Réseau d'aide aux personnes seules et itinérantes de Montréal (RAPSIM), site internet du RAPSIM, en ligne : RAPSIM < www.rapsim.org>. L'itinérance est un phénomène social qui se manifeste de façons variées et il est presque impossible de dégager un « profil type » de personne itinérante ou de personne à risque de le devenir : Voir Suzanne Bouclin, «Street Law’s Sites, Sights and Media » (2011) thèse de doctorat en droit civil, Université McGill, 2011, non publiée [Bouclin 2011].

25 Pour une introduction au concept voir : Karim Benyekhlef et Fabien Gélinas (avec la collaboration de Rémy Khouzam) Le règlement en ligne des conflits : enjeux de la cyberjustice, Romillat, Paris, 2003. 
TIC dans le processus judiciaire et en mettant à la disposition des gens des outils de simplification et de vulgarisation des procédures judiciaires et du droit; et intégrer les TIC dans la pratique judiciaire quotidienne $^{26}$.

D'abord, afin d'améliorer l'accessibilité à la justice, les chercheurs du Laboratoire de cyberjustice de Montréal (" Laboratoire de cyberjustice ») ${ }^{27}$, dont l'objectif est de développer des solutions technologiques aux problèmes auxquels est confronté le système judiciaire ${ }^{28}$, travaillent en ce moment à une application de gestion et de dépôt électronique des procédures ( «e-filing»), à une application de gestion des échéanciers et du calendrier ainsi qu'à une application sur le règlement en ligne de litige $[R L L]^{29}$. De telles applications font déjà leur apparition un peu partout dans le monde ${ }^{30}$. En Europe, des applications comme celles que souhaite développer le Laboratoire de cyberjustice existent depuis $2010^{31}$. L’Union européenne a ainsi instauré un " portail e-justice » ${ }^{32}$, traduit en 22 langues, qui vise à aider tant les citoyens que les entreprises, les avocats et les juges qui s'intéressent au système de justice d'autres États européens ${ }^{33}$. On y trouve des informations sur les procédures judiciaires des États membres et certains formulaires ${ }^{34}$. Au début de 2016, l’Union européenne aura mis en ligne une plateforme européenne de RLL entre consommateurs et commerçants dans le contexte des transactions en ligne ${ }^{35}$ qui établira un lien entre les parties en conflit et toutes les entités de règlement extrajudiciaire des litiges [REL] dans toutes les langues de l'Union ${ }^{36}$. La Commission des Nations Unies pour le droit

26 Les catégories que nous avons créées s’apparentent à celles qu’a établies Pierre-Claude Lafond, mais intègrent davantage d’outils issus de la cyberjustice, Pierre-Claude Lafond, L'accès à la justice civile au Québec : Portrait général, $3^{\mathrm{e}}$ éd, Cowansville, Yvon Blais, 2012 à la p 159 (« [c]ette accessibilité que favorise la technologie se remarque à deux manifestations : le développement de modes de règlement des conflits en ligne et la cyberjustice au service des tribunaux »).

27 Laboratoire de cyberjustice, « Découvrez le Laboratoire de cyberjustice en 8 min » (juillet 2013), en ligne : Laboratoire de cyberjustice <http://www.laboratoiredecyberjustice.org/fr/Home/Home> [Laboratoire de cyberjustice].

28 Centre de recherche en droit public, « Le Laboratoire sur la cyberjustice : Repenser le processus judiciaire » (non daté), en ligne : CRDP <http://www.crdp.umontreal.ca/fr/activites/information/165.html>.

29 En anglais, ODR pour « Online Dispute Resolution ». Pour bien comprendre le mode de fonctionnement des plateformes RLL, voir Benyekhlef et Gélinas, supra note 25 aux pp 65-66, 75, 77. Pour un point de vue critique du RLL issu d'une perspective académique et pratique : Martin Gramatikov, dir, Cost and Quality of Online Dispute Resolution. A Handbook for Measuring the Costs and Quality of ODR, Maklu Publishers, 2012. Le RLL permet plusieurs avantages dans le contexte de la résolution des différends, incluant la flexibilité, la rapidité, la spécificité, le coût réduit, la confidentialité et la sauvegarde potentielle des relations futures, voir Alexia Roussos, "La résolution des différends », en ligne : (2000) 6 :1 Lex Electronica <http://www.lex-electronica.org/docs/articles_167.htm>.

30 En Europe, il existe plus de 750 plateformes de règlement extrajudiciaire des litiges dont certaines offrent un processus complet en ligne : European Commission, « ADR and ODR in the EU today » (juillet 2013), en ligne : European Commission <http://ec.europa.eu/consumers/redress_cons/adr_odr_eu_en.htm>.

31 Par applications, on entend les programmes destinés à aider l'utilisateur d'un ordinateur : Larousse, 2012, sub verbo « application ».

32 Voir le Portail e-justice européen, en ligne : Portail e-justice européen <https://e-justice.europa.eu> [Portail e-justice].

33 Portail e-justice, ibid.

34 Pour en avoir quelques exemples, voir Portail e-justice européen, Formulaires dynamiques, en ligne : <https://ejustice.europa.eu/content_dynamic_forms-155-fr.do>.

35 European Commission, « New legislation on Alternative and Online Dispute Resolution (ADR) and (ODR) » (29 novembre 2011), en ligne : European Commission <http://ec.europa.eu/consumers/redress_cons/adr_policy_work_en.htm>.

36 Ibid. 
commercial international [CNUDCI] a, quant à elle, un groupe de travail qui se penche présentement sur le règlement en ligne de litige ${ }^{37}$. Le Hague Institute for the Internationalization of Law travaille pour sa part à l'évolution d'une plateforme web, déjà fonctionnelle, exploitée par l'aide juridique néerlandaise qui accompagne, étape par étape, les individus dans leur processus de résolution de conflit ${ }^{38}$. Le site actuel pose plusieurs questions à l'utilisateur et lui propose des pistes de solution ou de médiation, ainsi que des outils utiles à la résolution de son problème ${ }^{39}$. Le projet «Rechtwijzer 2.0 » souhaite internationaliser ce site web et élargir son mandat ${ }^{40}$. Plusieurs pays ont également des initiatives prises en ce sens ${ }^{41}$. Aux Pays-Bas, un processus de médiation en ligne en matière de divorce a donné de bons résultats quant à la satisfaction des parties ${ }^{42}$ (dans un contexte que nous présumons consensuel et dont la violence conjugale est absente ${ }^{43}$ ). Le Centre canadien de technologie judiciaire promeut de son côté la modernisation des services judiciaires, entre autres en construisant des bases de données accessibles et en offrant des conseils aux avocats sur l'utilisation des nouvelles technologies ${ }^{44}$.

Ensuite, le Laboratoire de cyberjustice a formulé quelques recommandations afin que l’intégration

37 Commission des Nations Unies pour le droit commercial international, Groupe de Travail III, « 2010 à ce jour :

Règlement des litiges en ligne » (2013), en ligne : Commission des Nations Unies pour le droit commercial international $<$ http://www.uncitral.org/uncitral/commission/working_groups/3Online_Dispute_Resolution.html>.

Hague Institute for the Internationalization of Law, «Interactive platform to justice: Rechtwijzer 2.0 », en ligne : Hague Institute for the Internationalization of Law < http://www.hiil.org/pro-ject/rechtwijzer> [Hague Institute].

39 Ibid.

40 Ibid.

41 Pour en avoir quelques exemples, voir Commission des Nations Unies pour le droit commercial international, « Online Dispute Resolution: On-line Resources » (2013), en ligne : Commission des Nations Unies pour le droit commercial international < http://www.uncitral.org/uncitral/en/publications/online_resources_ODR.html>. Il est également pertinent de noter que l’Organisation des États Américains a proposé aux Nations Unies en 2006 des lignes directrices quant à l'adoption d'un modèle RLL, voir Organisation des États Américains, « Building a Practical Framework for Consumer Protection » (juillet 2013), en ligne : Organisation des États Américains <http://www.oas.org/dil/CIDIPVII_consumer_protection_united_states_presentation.pdf $>$.

42 Martin Gramatikov et Laura Klaming, « Getting divorced online: Procedural and Outcome Justice in Online Divorce Mediation », février 2011 [non publié], archivé en ligne: Social Science Research Network <http://papers.ssrn.com/sol3/papers.cfm?abstract_id=1752903>. Ce projet est également chapeauté par le Hague Institute for the Internationalization of Law: Hague Institute for the Internationalization of Law, « Divorce online », en ligne: Hague Institute for the Internationalization of Law <http://www.hiil.org/project/divorce-online>.

43 Selon Trina Grillo, la médiation, qui traite le conflit en prenant pour acquis que les parties sont à égalité, peut avoir des conséquences néfastes pour les femmes, qui ne sont pas historiquement sur le même pied d'égalité que les hommes, Trina Grillo, « The Mediation Alternative : Process Dangers for Women » (1991) 100:6 Yale LJ 1545. Les théories sur la médiation indiquent qu’elle ne fonctionne réellement que dans un contexte consensuel et où il n’y a pas de violence entre les parties, voir Renu Mandhane, «The trend towards mandatory mediation: A critical feminist legal perspective » (août 1999), en ligne : Legal information on issues related to violence against women <http://owjn.org/owjn_2009/legal-information/aboriginal-law/161>; Charbonneau et al, Mediation in cases of domestic abuse: Helpful option or unacceptable risk? The final report of the Domestic Abuse and Mediation Project, Maine, Maine Court Mediation Service, 1992; Carol Smart, « Power and the Politics of Custody » dans Carol Smart et Selma Sevenhuijsen, dir, Child Custody and the Politics of Gender, New York, Routledge, 1989, 1; Jessica Pearson, « Mediating when domestic violence is a factor: Policies and practices in court-based divorce mediation programs » (1997) 14:4 Mediation Quarterly, 319.

44 Site internet du Centre canadien de technologie judiciaire, en ligne : Centre canadien de technologie judiciaire, $<$ http://ccct-cctj.ca/>. 
des TIC dans la pratique judiciaire soit réussie ${ }^{45}$; il recommande ainsi de déterminer clairement les répercussions possibles de l'intégration des TIC sur les comportements humains et les pratiques judiciaires, de cerner les besoins réels des individus les plus touchés par cette intégration et de promouvoir une approche collaborative et interdisciplinaire ${ }^{46}$.

Des collaborateurs du Laboratoire ont également tenté de cerner les risques légaux associés à la cyberjustice $^{47}$. Selon eux, le risque principal se situe au niveau des principes de base de notre système de justice - telles que les garanties constitutionnelles -, qui pourraient souffrir des effets et des changements engendrés par la cyberjustice ${ }^{48}$. Fabien Gélinas a pour sa part exploré les problèmes informatiques liés à la cyberjustice, notamment l'interopérabilité des systèmes et la modélisation ${ }^{49}$. D'autres soulignent comment la cyberjustice pourrait favoriser l'échange d'informations, la participation des collectivités et le contact avec celles-ci dans le contexte judiciaire émergent de la justice transitionnelle $e^{50}$.

Plusieurs auteurs cherchent également à voir comment la vidéoconférence ${ }^{51}$ a été intégrée dans la pratique judiciaire quotidienne. C'est le cas d'Anne Wallace, qui s'est intéressée à l'arrivée de la visioconférence judiciaire dans les régions éloignées et mal desservies de l'Australie, principalement habitées par des populations autochtones. La vidéoconférence permet ainsi certaines procédures judiciaires sans que les justiciables n'aient à se déplacer physiquement. ${ }^{52}$ Cette implantation diminue les coûts habituellement élevés du maintien d'un système de justice dans ces régions, notamment parce que le juge et les avocats n'ont pas à se déplacer, et réduit les délais associés au système judiciaire ${ }^{53}$. Cette technologie est également perçue comme une façon d'amener divers services sociaux à la communauté, par exemple pour gérer les problèmes de violence conjugale ${ }^{54}$. Elle permet aussi à des personnes habitant des régions éloignées d'obtenir les services d'un avocat à distance ${ }^{55}$. L'auteure souligne toutefois que cette façon de faire n'est pas sans soulever des difficultés dans le contexte autochtone, les

45 Nicolas W. Vermeys et Karim Benyekhlef, « Best Practices in the Field of Cyberjustice » (avril 2011), en ligne : IIJusticia <http://www.iijusticia.org/docs/Karim\%20Nicolas.pdf > [Vermeys et Benyekhlef].

$46 \quad$ Ibid aux pp 2, 7 et 11.

47 François Sénécal et Karim Benyekhlef, « Groundwork for Assessing the Legal Risks of Cyberjustice » (2009) 7:1 CJLT 41 [Sénécal et Benyekhlef].

48 Ibid à la p 50.

49 Fabien Gélinas, «Interopérabilité et normalisation des systèmes de cyberjustice : Orientations », en ligne : (2006) 10:3 Lex Electronica <http://www.lex-electronica.org/docs/articles_62.pdf> aux pp 4-6 et 10 [Gélinas 2006].

50 Philipp Kastner, « Cyberjustice in the Context of Transitional Justice » (novembre 2013), en ligne : Laboratoire de cyberjustice <http://site.cyberjustice.ca/Content/documents/WP009_TransitionnalJusticeAndCyberjustice_en.pdf> aux pp 9-10.

51 Les termes vidéoconférence et visioconférence correspondent à « video conferencing » en anglais. Pour en avoir quelques exemples, voir Cléa Iavarone-Turcotte et Astrid Daigneault-Guimond, « Bibliographie commentée » (4 mai 2011), en ligne : Laboratoire de cyberjustice $<$ http://www.laboratoiredecyberjustice.org/Content/documents/bibliographie_commentee.pdf> aux pp 126 et ss.

52 Anne Wallace, «'Virtual Justice in the Bush': The Use of Court Technology in Remote and Regional Australia » (2008) 19 J L \& Info Sci 1 [Wallace].

53 Ibid aux pp 3, 12.

$54 \quad$ Ibid à la $\mathrm{p} 11$.

55 Suzie Forell, Meg Laufer et Erol Digiusto, « Legal assistance by videoconferencing: what is known » (2011) nov 2011: paper 15 Law and Justice Foundation: Justice Issues

<http://www.lawfoundation.net.au/ljf/app/\&id=B0A936D88AF64726CA25796600008A3A>. 
rapports entre le système de justice étatique et les populations autochtones étant toujours marqués par une histoire de colonialisme, d'aliénation et de racisme. Ces populations pourraient se sentir encore plus méfiantes face à un système de justice préférant les rapports dématérialisés - par écrans interposés - aux rencontres en personne. Cette méfiance ne pourra que se trouver exacerbée par le fait que ces systèmes soient installés dans les postes de police. ${ }^{56}$ De manière générale, le choix de cet emplacement devrait soulever des doutes sur l'indépendance du système de justice vis-à-vis des forces de l'ordre. De plus, dans le cas particulier des populations autochtones, s'ajoute à cette inquiétude une relation tendue avec le corps policier. ${ }^{57}$ En effet, ce dernier a souvent appuyé l'État dans ces entreprises coloniales ${ }^{58}$ et continue d'être perçu comme agissant dans l'intérêt de l'État plutôt que dans celui des populations autochtones - notamment parce qu'il est l'agent premier de l'application d'un droit étatique qui manque souvent de légitimité aux yeux de ces dernières ${ }^{59}$. De plus, l'utilisation de la technologie ne règle en rien les problèmes déjà existants en ce qui concerne l'application du droit étatique aux peuples autochtones. Ces problèmes sont de plusieurs ordres et nous n'avons pas la prétention d'en faire ici le relevé exhaustif. Soulignons cependant que - outre la légitimité nécessairement problématique qu’un système de droit imposé unilatéralement et souvent par la force ${ }^{60}$ - les rapports entre les peuples autochtones et le droit étatique s'avèrent difficiles notamment pour des raisons linguistiques et culturelles. Ainsi, les langues autochtones sont fondamentalement différentes de celles d'origines européennes utilisées dans les tribunaux étatiques. ${ }^{61}$ Les services d'interprètes ne suffisent pas à traduire les concepts et les visions du monde distincts que chaque langue sert à exprimer. Par ailleurs, les principes autochtones qui soustendent les processus de résolution des conflits sont souvent différents de ceux qui fondent les processus étatiques, et sont parfois même incompatibles avec ceux-ci ${ }^{62}$.

Un projet de justice par vidéoconférence similaire est en cours de développement dans plusieurs régions du Québec et dans le reste du Canada, notamment à Amos, siège de la Cour itinérante au Québec $^{63}$. Ce système permet présentement aux prévenus du Nunavik d’obtenir leur enquête sur remise

56 Les auteurs Pierrot Ross-Tremblay et Nawel Hamidi soulignent ainsi l'importance de rappeler «les fondements colonialistes et ethnocentristes qui imprègnent toutes les relations de la Couronne avec les Premiers peuples du Canada» . Pierrot Ross-Tremblay et Nawel Hamidi, « Les écueils de l'extinction. Les Premiers peuples, les négociations territoriales et l'esquisse d'une ère postcoloniale » (2013) 43 :1 Recherches amérindiennes au Québec à la p. 52

57 Suzie Forell, Meg Laufer et Erol Digiusto à la p 16.

58 Sur l'importance de la contribution des forces policières aux processus coloniaux en Australie et au Canada, voir notamment Amanda Nettelbeck and Russell Smandych, « Policing Indigenous Peoples on Two Colonial Frontiers: Australia's Mounted Police and Canada's North-West Mounted Police» (2010) 43 Australian \& New Zealand Journal of Criminology 356.

59 Ibid à la p 16. Voir aussi Elizabeth Comack, Racialized Policing : Aboriginal People's Encounters with the Police, Winnipeg, Fernwood Publishing, 2012.

60 Pour une discussion détaillée sur la manière dont le droit étatique d’origine européenne a été imposé aux Premières nations au Canada, voir notamment Sébastien Grammond, Aménager la coexistence. Les peuples autochtones et le droit canadien, Yvon Blais-Bruylant, Bruxelles, 2003 aux p. 70-101.

61 Suzie Forell, Meg Laufer et Erol Digiusto à la p 14.

62 Ibid à la p 14. Voir aussi : John Borrows, «With or Without You: First Nations Law in Canada» (1996), McGill Law Journal [(1996) 41 McGill L.J. 629], pp. 629 à 635.

63 Cour du Québec : Rapport public 2012, Bureau de la juge en chef du Québec, 2013 à la p 26; Sur l'utilité de la vidéoconférence dans le domaine du droit matrimonial et familial, voir notamment Ministère de la Justice, « Use of technology in Family Law Matters » (26 avril 2013), en ligne : justice.gc.ca <http://www.justice.gc.ca/fra/df-fl/sjffjs/affic-view.asp?uid=500>. 
en liberté à distance ${ }^{64}$ et donc plus rapidement : grâce à la vidéoconférence, le juge, le procureur de la poursuite, le prévenu et l'avocat de la défense n’ayant pas à se trouver physiquement dans le même lieu $^{65}$. En l'absence de ce système, lorsqu'un juge de paix ordonne la détention d'un individu du Grand Nord québécois par suite de la commission d'un crime ${ }^{66}$, l'accusé doit, sauf exception, être déplacé par avion et autobus jusqu'à la prison d'Amos ${ }^{67}$. Le détenu subit donc son enquête sur remise en liberté au palais de justice d'Amos. Avant l'instauration du système de vidéoconférence, le délai maximal de trois jours prévu au Code criminel $^{68}$ et précédant la tenue de l'enquête sur remise en liberté était souvent dépassé à cause de la conjugaison du temps de déplacement par avion et des éléments suivants : à Amos, le juge n'était alors disponible qu'une seule journée par semaine pour tenir l'enquête sur remise en liberté; et la majorité des avocats de la défense des accusés du Nunavik habitent en dehors de la région d'Amos $^{69}$. Grâce au système de visioconférence, des juges sont désormais disponibles quatre jours par semaine pour tenir les enquêtes sur remise en liberté; ces juges proviennent d'autres villes qu'Amos et siègent par visioconférence ${ }^{70}$. L'avocat de la défense peut quant à lui participer à la visioconférence à partir du palais de justice de sa ville de résidence ou, tout simplement, à partir de sa résidence personnelle $^{71}$. L'objectif de la Cour du Québec est de faire en sorte qu'éventuellement, le détenu luimême n'ait plus à se déplacer jusqu'à Amos et puisse obtenir son enquête sur remise en liberté par visioconférence à partir de sa communauté ${ }^{72}$. Nous n'avons trouvé aucune recherche qui évalue l'impact de la vidéoconférence sur les prévenus, mais nous pouvons inférer que, la vidéoconférence étant installée pour servir les résidents des communautés nordiques comportant une grande concentration d'autochtones, certaines des préoccupations soulevées par Wallace s’appliqueraient également dans ce contexte $^{73}$.

64 L'Association du Barreau canadien vient de publier un rapport sur l'utilisation de la technologie en matière pénale. Elle recommande entre autres d'inclure dans le Code criminel une disposition permettant au juge de tenir une audience de mise en liberté provisoire ou une enquête préliminaire par vidéoconférence dans certains contextes. Association du Barreau canadien « Version provisoire du Rapport sur l'usage de la technologie dans le système de justice pénale » (25 février 2013), en ligne : <http://www.cba.org/abc/memoires/PDF/13-11-fr.pdf>.

Pour l'instant, le procureur de la poursuite et le détenu se trouvent dans le même lieu lors de l'enquête sur remise en liberté ; pour leur part, le juge et l'avocat de la défense communiquent souvent par vidéoconférence à partir de lieux distincts. Entrevue de $\mathrm{M}^{\mathrm{e}}$ Marie-Josée Barry-Gosselin, procureure aux poursuites criminelles et pénales à la Cour itinérante, par $\mathrm{M}^{\mathrm{e}}$ Marie-Andrée Denis-Boileau, la chercheure émergente, (2 octobre 2013), correspondance conservée dans les dossiers de la chercheure [Entrevue de $\mathrm{M}^{\mathrm{e}}$ Barry-Gosselin]. La Cour du Québec planifie toutefois que l'accusé demeure éventuellement dans sa communauté et communique aussi par visioconférence, les quatre acteurs judiciaires se trouveront alors dans quatre endroits distincts lors de l'enquête sur remise en liberté, Cour du Québec : Rapport public 2012, ibid à la p 26.

66 Lors de la comparution du détenu devant un juge de paix, Code criminel, LRC 1985, c C-46, art 503.

67 Entrevue de $\mathrm{M}^{\mathrm{e}}$ Barry-Gosselin, supra note 65.

68 Code criminel, LRC 1985, c C-46, art 516(1).

69 Entrevue de $\mathrm{M}^{\mathrm{e}}$ Barry-Gosselin, supra note 65.

70 Ibid.

71 Ibid.

72 Cour du Québec: Rapport public 2012, supra note 63.

73 Cour du Québec : Rapport public 2012, supra note 63 à la p 26. Nous ne croyons pas que la solution aux problèmes des distances, des délais et de l'engorgement des rôles soit la tenue d'une enquête sur remise en liberté par visioconférence où il est possible qu'aucun des quatre participants ne se trouve dans la même ville (par exemple, le juge peut être à Val 
Les tablettes numériques attirent également l'attention des juristes. Luigi Benetton, un commentateur du milieu juridique, présente les applications du iPad@ comme étant utiles aux avocats pour la recherche légale, les procédures et la documentation ${ }^{74}$. Le Laboratoire de cyberjustice de Montréal intègre actuellement ces tablettes dans ses travaux ${ }^{75}$. Ses membres ont en outre l'ambition d'intégrer la technologie holographique à leurs projets, par exemple pour permettre la reconstruction d'une scène de crime ou le témoignage à distance ${ }^{76}$.

L’intégration des médias sociaux dans la pratique judiciaire quotidienne intéresse aussi certains juristes. Pamela Schulz mentionne la présence marquée des médias sociaux dans la sphère publique actuelle $^{77}$. Elle souligne l'importance pour les tribunaux d'être présents dans les médias sociaux pour s'assurer le contrôle de leur discours ${ }^{78}$. Les magistratures anglaise et écossaise sont peut-être du même avis : depuis respectivement 2011 et 2010, elles ont un compte Twitter $($ : des nouvelles sur le travail des juges et magistrats ainsi que des tribunaux de Grande-Bretagne y sont " gazouillées » ${ }^{79}$. La Cour suprême du Canada, quant à elle, est inscrite sur Facebook@ depuis le 2 février 2011, mais n’y a toujours rien publié ${ }^{80}$. L'expérience des tribunaux de l'État de New York sur Twitter semble prouver un intérêt pour les communications directes venant des tribunaux: leur nombre d'abonnés Twitter a augmenté de 52 p. 100 entre 2011 et $2012^{81}$. Leur chaîne YouTube a quant à elle vu augmenter de 140 p. 100 son nombre de visiteurs pendant cette même période ${ }^{82}$. Partout au Canada, certains tribunaux sont présents sur les médias sociaux, mais ils constituent une minorité. Les tribunaux de la Nouvelle-Écosse ont tous un compte Twitter sur lequel ils diffusent leurs décisions, et quatre comptes ayant des objectifs spécifiques (nouvelles de la Cour, avis pour les professionnels du droit, modifications aux règles de procédure civile et changements aux rôles en ligne) ont été créés par les tribunaux de la province $^{83}$. Au Québec, seule la Cour du Québec est présente sur Twitter ${ }^{84}$. Elle y diffuse principalement

d’Or, le procureur, à Amos, le prévenu, dans une communauté du Nunavik et l'avocat de la défense, à Montréal ou ailleurs au Québec).

74 Luigi Benetton, « iPad apps for lawyers » (2013), en ligne : L’Association du Barreau canadien

$<$ http://www.cba.org/CBA/practicelink/careerbuilders_technology/iPad.aspx>.

75 Laboratoire de cyberjustice, supra note 27.

76 Ibid.

77 Pamela Schulz, « Trial by Tweet? Social media innovation or degradation? The future and challenge of change for courts » (2012) 22 JJA 29.

78 Ibid à la p 36.

79 Compte Twitter de la magistrature anglaise, en ligne : Twitter <https://twitter.com/JudiciaryUK>; Compte Twitter de la magistrature écossaise, en_ligne : Twitter < https://twitter.com/JudgesScotland $>$.

80 Compte Facebook francophone de la Cour suprême du Canada, en ligne : Facebook <https://www.facebook.com/coursupremeducanada>; Compte Facebook anglophone de la Cour suprême du Canada, en ligne : Facebook <https://www.facebook.com/supremecourtofcanada>. Selon Mary-Rose Papandrea, ce fait ne découle pas nécessairement d'une technophobie ou d'une ignorance mais plutôt d'une préoccupation de la cour quant à la culture médiatique moderne : Mary-Rose Papandrea, « Moving beyond cameras in the courtroom: the supreme court, technology and the media » (2013) 2012:6 BYUL Rev 1901 à la p 1903.

81 New York State Courts Access to Justice Program (2012), 2012 Report, en ligne : NYcourts <http://www.nycourts.gov/ip/nya2j/pdfs/NYA2J_2012report.pdf> à la p 38.

82 Ibid à la p 37.

83 The Courts of Nova Scotia, «Courts of Nova Scotia Enter The "Twitterverse" », février 2012, en ligne : The Courts of Nova Scotia <http://www.courts.ns.ca/twitter.htm>.

84 Compte Twitter de la Cour du Québec, en ligne : Twitter <https://twitter.com/Cour_du_Quebec>. 
des félicitations et des informations sur ses activités (participation de la Cour à divers évènements, rentrées judiciaires, publications de rapports, etc.), mais pas sur ses décisions ${ }^{85}$.

En résumé, au Canada, mis à part en Nouvelle-Écosse, les différents tribunaux ne manifestent pas de réel intérêt pour l'utilisation des médias sociaux comme moyen de communiquer avec la population. L'Institut canadien d'information juridique (CanLII) a toutefois créé des comptes Twitter sur lesquels il diffuse les décisions judiciaires rendues par les différentes cours d’appel du pays et par la Cour suprême du Canada ${ }^{86}$. Quant à l'utilisation des médias sociaux en salle d'audience, le bureau du plus haut juge de Grande-Bretagne, le Lord Juge en chef de l'Angleterre et du Pays de Galles, a fourni en 2011 des instructions sur l'utilisation des communications écrites synchrones (incluant Twitter) en salle d'audience ${ }^{87}$. On y a réitéré l'interdiction d'enregistrements audio, de photographies ou du téléphone, en précisant toutefois qu'il est possible de communiquer par texte, tant que cette communication reste silencieuse. Du coup, la messagerie instantanée, Twitter ou toute forme de blog, peut être utilisée en salle d'audience. Au Canada, aucune directive ne lie l'ensemble des tribunaux du pays : chaque tribunal est libre de traiter de cette situation comme il l'entend ${ }^{88}$. Le Conseil canadien de la magistrature

85 Ibid

86 Simon Fodden, « CanCourts », en ligne: CanCourts <http://www.cancourts.ca> [Fodden].

87 The Lord Chief Justice of England and Wales, « Practice guidance: The use of live text-based forms of communication (including Twitter) from court for the purpose of fair and accurate reporting » (14 décembre 2011), en ligne:

judiciary.gov.uk <http://www.judiciary.gov.uk/Resour-ces/JCO/Documents/Guidance/ltbc-guidance-dec-2011.pdf>.

88 Politiques des différents tribunaux du Canada, d'Ouest en Est (les tribunaux de Terre-Neuve n'ont aucune politique à ce sujet, la plupart des tribunaux des Territoires du Nord-Ouest non plus; pour voir la politique québécoise, infra note 92; pour la politique de la Cour suprême, infra note 91) : Nunavut Court of Justice, « Media use of technology in the courtroom and courthouse $», 1^{\text {er }}$ avril 2013, en ligne : nucj.ca

<http://www.nucj.ca/rules/Media_TechnologyUse_Courtrooms.pdf>; Nunavut Court of Justice, « Public use of technology in the courtroom and courthouse ", $1^{\mathrm{er}}$ avril 2013, en ligne : nucj.ca

<http://www.nucj.ca/rules/Public_TechnologyUse_Courtrooms.pdf>; Territorial Court of the Northwest Territories, " Interim policy: Use of electronic devices in the courtroom », 23 juillet 2012, en ligne : nwtcourts.ca $<$ https://www.nwtcourts.ca/directives/PD-TCa.pdf> [Territorial Court of NWT]; Cour territoriale du Yukon, « Directive de pratique $\mathrm{n}^{\circ} 25$ : Utilisation de matériel d'enregistrement et d’équipement électronique lors d’instances judiciaires », 7 mai 2013, en ligne : yukoncourts.ca <http://www.yukoncourts.ca/fr/pdf/P-D_25_Electronic_Devices-FR_.pdf >; Cour suprême du Yukon, « Directive de pratique $n^{\circ} 60$ : Utilisation de matériel d'enregistrement et d'équipement électronique lors d'instances judiciaires », 7 mai 2013, en ligne : yukoncourts.ca <http://www.yukoncourts.ca/fr/pdf/pd_60_utilisation_de_material_denregistrement_.pdf>; Court of Appeal of British Columbia, « Policy on use of electronic devices in courtrooms », 17 septembre 2012, en ligne : Courts.gov.bc.ca $<$ http://www.courts.gov.bc.ca/supreme_court/media/PDF/Policy\%20on\%20Use\%20of\%20Electronic\%20Devices\%20in\%20Courtrooms\%20-\%20FINAL.pdf>; Court of Queen’s Bench of Alberta, « Electronic and Wireless Devices Policy », janvier 2012, en ligne : Alberta courts $<$ http://www.albertacourts.ab.ca/qb/Electronic_Policy_FINAL.pdf>; Saskatchewan Law Courts, « Twitter and other Text-Based Forms of Media Communication from Saskatchewan Courtrooms », 2012, en ligne : Saskatchewan Law Courts <http://www.sask-lawcourts.ca/images/documents/Resources/Twitter_Protocol_2012.pdf>; Manitoba Courts, « Electronic Devices Policy », $1^{\mathrm{er}}$ septembre 2013, en ligne : <http://www.manitobacourts.mb.ca/pdf/electronic_device_policy.pdf $>$; Cour de justice de l’Ontario, « Protocole sur l'utilisation de dispositifs de communication électroniques dans la salle d'audience », $1^{\mathrm{er}}$ mars 2013, en ligne : Ontario courts <http://www.ontariocourts.ca/ocj/fr/avocats/directives-de-pratique/communication-electroniques/>; Cour supérieure de justice de l’Ontario, « Protocole sur l'utilisation de dispositifs électroniques dans la salle d'audience », $1^{\mathrm{er}}$ février 2013, en ligne : Ontario Courts <http://www.ontariocourts.ca/scj/fr/pratique/directives-de-pratique/dispositifselectroniques/\#4_Utilisation_de_dispositifs_lectroniques_dans_la_salle_d8217audience>; Cour d'appel, Cour du Banc 
recommande simplement que les tribunaux élaborent des politiques à ce sujet ${ }^{89}$. La position des différents tribunaux est donc hétéroclite; toutefois, la plupart d'entre eux permettent l'utilisation d'appareils électroniques en salle d'audience, pour communiquer avec l'extérieur de la salle, tant que ces communications ne dérangent pas le processus judiciaire ${ }^{90}$. Par exemple, la Cour suprême du Canada permet l'utilisation des ordinateurs et appareils de poche (comme les téléphones intelligents) en salle d'audience et fournit même aux médias un accès sans fil à Internet et des prises de courant ${ }^{91}$. Le Québec est la seule province où il est interdit aux médias et à quiconque d'utiliser un appareil électronique dans le but de diffuser ou de communiquer quelque information que ce soit à partir de la salle d'audience vers l'extérieur ${ }^{92}$; la même interdiction s'applique à la cour territoriale des Territoires du Nord-Ouest ${ }^{93}$. En 2012, le Juge-président principal et le Premier président des Tribunaux administratifs de l'Angleterre et du Pays de Galles en Grande-Bretagne ont communiqué des directives à la magistrature concernant l'utilisation des blogs (ces directives incluent l'utilisation de Twitter) ${ }^{94}$. On y fait mention de l'importance de la confiance du public en l'impartialité des juges ${ }^{95}$. Il n'est pas interdit à ceux-ci d'utiliser des blogs, mais ils ne peuvent s'y présenter comme étant membres de la magistrature $^{96}$. Ils ne doivent pas non plus émettre des opinions qui pourraient ébranler la confiance du public en leur impartialité ou en celle de la magistrature en général. Aux États-Unis, les tribunaux de l'État de New York («New York State Courts ») ont une chaîne YouTube ${ }^{97}$ sur laquelle ils diffusent des informations sur le fonctionnement du tribunal, certaines vidéos se présentant même sous forme de « do it yourself ${ }^{98}$. Ces tribunaux disposent également d'un compte Twitter qui leur permet de diffuser des informations dans le but d'assurer l'accès à la justice à tous les New Yorkais ${ }^{99}$.

de la Reine et Cour provinciale du Nouveau-Brunswick, « Directive respecting electronic devices in the courtroom », 29 juin 2012; The Courts of Nova Scotia, « Use of Electronic Devices in Nova Scotia Courthouses », 29 avril 2013 , en ligne : Courts.ns.ca <http://www.courts.ns.ca/media_access/electronic_devices_policy_08_03.htm>; Prince Edward Island Court of Appeal, « Practice Directions », 18 mars 2011, en ligne : Gov.pe.ca

$<$ http://www.gov.pe.ca/courts/supreme/PracticeDirections.pdf> aux pp 26-27.

89 Conseil canadien de la magistrature, Cadre de politique de gestion de l'information judiciaire dans le monde numérique, 2013 [non publié], archivé en ligne : Conseil canadien de la magistrature, <http://www.cjcccm.gc.ca/cmslib/general/AJC/Information\%20Judiciaire\%20dans\%20le\%20monde\%20numérique\%202013-03.pdf> à la p 46.

90 Supra note 88.

91 Cour suprême du Canada, « Accès à la Cour », 2009, en ligne : Cour suprême du Canada <http://www.scccsc.gc.ca/news-nouv/media/acc-fra.aspx>.

92 Cour d’appel du Québec, « Lignes directrices concernant l'utilisation des technologies en salle d'audience », 15 avril 2013, en ligne : Tribunaux.qc.ca <http://www.tribunaux.qc.ca/cappel/Actualite/Quoideneuf/docs/dir_utilisation_techno_salle_audio.pdf>.

93 Territorial Court of NWT, supra note 88.

94 Senior Presiding Judge for England and Wales et Senior President of Tribunals, « Blogging by Judicial Office Holders » (août 2012), en ligne: judiciary.gov.uk <http://www.judiciary.gov.u-k/Resources/JCO/Documents/Guidance/bloggingguidance-august-2012.pdf>.

95 Ibid.

96 Ibid.

97 Chaîne Youtube de « access to justice NY state courts », en_ligne : Youtube <http://www.youtube.com/user/NYCourtsA2J?feature $=$ watch $>$.

98 Traduction libre : « Faites-le vous-même ». « Do it yourself », ou DIY est une expression consacrée sur la toile, voir Urban Dictionary, sub verbo « DIY », en ligne : <http://www.urbandictionary.com/define.php?term=DIY>.

99 Compte Twitter de « access to justice NY state courts », en_ligne : Twitter <https://twitter.com/NYCourtsA2J $>$. 
La cyberjustice inclut également les processus juridiques informels et offre de nouvelles façons d'accéder à l'information grâce à plusieurs médias qui facilitent l'autogestion juridique, tels des sites internet, blogs, services à écran tactile et vidéos ${ }^{100}$.

Cassandra Burke Robertson explique comment l'avènement des médias sociaux, et de Facebook en particulier, transformera le droit et le rendra plus accessible ${ }^{101}$. Selon elle, ces changements toucheront d'abord les citoyens avant de toucher les avocats ${ }^{102}$. Tout d'abord, en utilisant Facebook, une personne se représentant elle-même peut recueillir de l'information sur des témoins d'une manière qui n'est pas possible pour un avocat du point de vue éthique ${ }^{103}$. Cette même personne a également accès instantanément à un vaste public grâce au «crowdsourcing » qui, par un seul appel à tous, lui permet de consulter un grand nombre de personnes, certaines pouvant être avocates et prêtes à donner des conseils, d'autres ayant peut-être déjà eu une expérience pertinente qui leur permet d'aider la personne qui sollicite leur avis (par exemple, dans le cas d'un divorce $)^{104}$. Internet donne également accès à des services automatiques ou semi-automatiques d'assistance à la préparation de documents juridiques ${ }^{105} \mathrm{de}$ même qu'à des fournisseurs de services juridiques situés à l'étranger et offrant des services à moindre coût ${ }^{106}$.

Cette première section du travail avait pour but d'expliquer la cyberjustice. Nous en sommes venues à la conclusion que les formes de cyberjustice actuellement développées visent à améliorer l'accès à la justice de la population, principalement en intégrant les TIC dans le processus judiciaire et en mettant à la disposition des gens des outils de simplification et de vulgarisation des procédures judiciaires et du droit; elles visent également à intégrer les TIC dans la pratique judiciaire quotidienne. Bien que l’accès à la justice soit l'un des premiers buts visés par les chercheurs du domaine, nous nous sommes posé la question de savoir si ces différentes formes de cyberjustice pourraient potentiellement améliorer l'accès à la justice des personnes itinérantes ? Et si oui, dans quels contextes ?

\section{TIC ET POPULATIONS MARGINALISÉES ET ITINÉRANTES}

Le lien entre l'accès au droit et à la justice et un «changement effectif des conditions de vies des personnes marginalisées » a longtemps été établi ${ }^{107}$. Peu de recherches par contre ont exploré la façon

100 Katherine Bladow et Joyce Raby, « Using Social Media to Support Self-Represented Litigants and Increase Access to Justice » (2011) National Centre for State Courts: Future trends in state courts 2011 35; Benyekhlef et Gélinas, supra note 25.

101 Cassandra Burke Robertson, « The Facebook Disruption: How Social Media May Transform Civil Litigation and Facilitate Access to Justice » (2012) 65 Ark L Rev 75.

102 Ibid à la p 1. Voir aussi : Susskind, supra note 7.

103 Par exemple en faisant une demande d'amitié Facebook à une partie au litige, supra note 101 à la p 82.

104 Ibid à la p 84.

105 Ibid à la p 87.

106 Ibid à la p 89.

107 Avocats Sans Frontières, « Comment l'accès à la justice aide à réduire la pauvreté » (2103), en ligne : Avocats Sans Frontières <http://www.asf.be/wp-content/uploads/2013/03/Acc\%C3\%A8s-\%C3\%A0-la-justice-etpauvret\%C3\%A9.pdf> à la p 3; voir aussi Gaudence Kubwimana, «Burundi : Sans assistance, les pauvres ne peuvent accéder à la justice » (25 juin 2012), en ligne : Toukimontreal.com <http://toukimontreal.com/actualites/2012/06/25/burundi-sans-assistance-les-pauvres-ne-peuvent-acceder-a-la-justice/>; Byron M. Sheldrick, Legal Working Paper Series on Legal Empowerment for Sustainable Development: Access to 
dont la cyberjustice peut avoir un effet sur les populations vulnérables ou démunies face au système de justice. Melina Buckley, avocate et consultante en matière de politiques juridiques, souligne l'importance de l'utilisation des TIC pour remplir pleinement le mandat d'accès à la justice de l'aide juridique $^{108}$ et accéder à toute la population, indépendamment du niveau de revenu ${ }^{109}$. Des chercheurs ont toutefois constaté que les personnes ayant une faible scolarité manquent d'habiletés pour faire des recherches efficaces et juger de la qualité des conseils trouvés en ligne ${ }^{110}$. Les sociologues Silvana Greco et Silvia E. Vecchi ont quant à elles démontré l'aspect dangereux, ou négatif, de l'accès par le Web aux décisions judiciaires ${ }^{111}$. Elles évoquent le danger de creuser le fossé entre les personnes vulnérables et le reste de la population en rendant facilement accessibles par Internet les décisions des tribunaux, décisions qui contiennent énormément de données personnelles sur des populations parfois vulnérables $^{112}$. Bien que les objectifs prédominants soient la transparence ainsi que l'accès à l'information judiciaire et sa publication, il n’y a présentement pas d'équilibre entre ces objectifs et la nécessité de protéger la vie privée et l'intimité des personnes ${ }^{113}$. Malgré ces quelques timides avancées, une étude approfondie de l'impact de la cyberjustice sur l'accès à la justice des personnes marginalisées reste à effectuer ${ }^{114}$.

Les personnes marginalisées du système de justice le sont pour des raisons situationnelles (par exemple à cause de leur statut de migrant ou leur travail précaire), pour des raisons systémiques (telles les personnes «racisées " ${ }^{115}$, les personnes autochtones, les personnes ayant une déficience, les personnes âgées, les personnes issues d'une minorité visible) ou en raison de circonstances personnelles (analphabétisme, résistances au système d'éducation inadapté, dépendances, les personnes qui sont sans emploi ou dont le statut est précaire, les parents monoparentaux, les personnes qui reçoivent une pension d'invalidité ou des prestations d'aide sociale). Elles peuvent aussi l'être de façon cyclique (comme les personnes qui quittent le milieu carcéral ou les personnes souffrant de problèmes de santé mentale). Les personnes itinérantes se retrouvent souvent au carrefour d'un ou de plusieurs de ces modes de marginalisation (situationnel, systémique, personnel ou cyclique) : il est donc peu surprenant que

Justice and Legal Empowerment as Vehicles of Poverty Alleviation: Governance Challenges to Linking Legal Structures to Social Change, Montreal, Centre de droit international et de développement durable, 2012.

108 Supra note 101 aux pp 71 et 78.

109 Melina Buckley, Atteindre l'égalité devant la justice : Une invitation à l'imagination et à l'action (août 2013), en ligne : Association du Barreau canadien < http://www.cba.org/abc/justicepourtout/secure_pdf/Equal-Justice-Report-fra.pdf> à la p 10 [Buckley 2013].

110 Catrina Denvir, Nigel J. Balmer et Pascoe Pleasence, « Surfing the web - Recreation or resource? Exploring how young people in the UK use the Internet as an advice portal for problems with a legal dimension » (2011) 23:1 Interacting with Computers 96.

111 Silvana Greco et Silvia E.Vecchi, « Acciones participativas en el campo de la administración de justicia y tecnologías de información y comunicación (TIC) » dans Carlos G. Gregorio et Sonia Navarro Solano, dir, Internet y systema judicial en América Latina - Reglas de Heredia, Buenos Aires, Ad-Hoc, 2004.

112 Ibid à la $\mathrm{p} 7$.

113 Ibid.

114 C’est en partie l’objectif de la présente recherche, dans le contexte spécifique des personnes itinérantes. L’auteure principale mènera ensuite une recherche empirique sur ce sujet afin d'examiner certaines des propositions informelles qui se trouvent dans ce texte.

115 Le concept de « racialisation » fait référence aux processus sociaux et identitaires par lesquels des groupes sont socialement construits, voir Jean-Luc Primon, « Ethnicisation, racisation, racialisation : une introduction » (2007) 6 Faire Savoirs. 
l'aliénation et l'exclusion auxquelles elles font face soient particulièrement criantes.

Cette marginalisation porte certains à croire que ces personnes sont incapables d'utiliser les TIC et n'ont pas de culture numérique. Toutefois, beaucoup d'entre elles se servent déjà des TIC pour créer et soutenir des relations interpersonnelles ${ }^{116}$. D'abord, il a déjà été démontré que les sans-abri utilisent Internet ${ }^{117}$ : deux études américaines en sont venues à la conclusion que près de la moitié des sans-abri avaient utilisé un ordinateur dans les derniers mois et que près de la moitié de ceux-ci l'avaient fait pour des raisons liées au travail ${ }^{118}$. La plupart ont affirmé ne pas avoir de problème à accéder aux ressources du Web ${ }^{119}$. Catrina Denvir et ses collègues démontrent même que, chez les jeunes adultes sans-abri, la technologie est utilisée de façon similaire à l'utilisation faite par la population en général ${ }^{120}$.

Au Canada et au Québec, les personnes itinérantes ont aussi développé des liens de collaboration avec des organismes à but non lucratif et des militants pour l'accès libre aux données tels que Homeless Nation ${ }^{121}$, Logement Insalubre ${ }^{122}$ et le Homeless $\mathrm{Hub}^{123}$, pour se trouver de l'emploi, pour promouvoir leur droit au logement, pour accéder à de l'information sur la santé, pour se constituer des identités virtuelles et pour créer de nouvelles formes et conditions d'interaction entre personnes vivant en situation d'itinérance ${ }^{124}$.

Dans ce contexte, nous nous poserons maintenant les questions suivantes: D'abord, la cyberjustice est-elle un bon outil pour lutter contre la crise de l'accès à l'information et aux services juridiques vécue par les personnes marginalisées, et, plus particulièrement, par les personnes itinérantes? Et, dans l'affirmative, les personnes itinérantes se serviraient-elles des TIC pour combler leurs besoins juridiques ?

116 Suzanne Bouclin, « Producing Legal Subjectivities through Online Media » dans Anna Poletti et Julie Rak, dir, Identity Technologies: Producing Online Selves, Madison, University of Wisconsin Press, 2014 (publication à venir) [Bouclin 2014]; Jeff Karabanow et Ted D. Naylor, « Being Hooked-Up: Exploring the Experiences of Street Youth with Information Communication Technology » dans Dianne E. Looker et Ted D. Naylor, dir, Digital Diversity: Youth Equity and Information Technology, Waterloo, Wilfrid Laurier University Press, 2010, 161 [Karabanow et Naylor]; Christopher A. LeDantec et W. Keith Edwards, « Designs on Dignity: Perceptions of Technology Use Among the Homeless » dans Desney Tan, dir, Proceedings of the 26th Annual SIGCHI Conference on Human Factors in Computing Systems, New York, ACM Press, 2008 627; Mary Anne Moser, « A Study of Computers in Homeless Shelters » (2008) 10 Sc. Tech \& Hum Values 393; Randal D. Pinkett, « The Creating Community Connections Project » dans Jonathan Lazar, dir, Universal Usability: Designing Computer Interfaces for Diverse User Populations, West Sussex, England, John Wiley and Sons, 2007 457; Mitchel Resnick, Natalie Rusk et Stina Coole, « The Computer Clubhouse: Technological Fluency in the Inner City » dans Donald A. Schön, Bish Sanyal et William J. Mitchell, dir, High Technology for Low-Income Communities, Cambridge, MIT Press, 2008263.

117 Karin M. Eyrich-Garg, « Sheltered in cyberspace? Computer use among the unsheltered 'street' homeless » (2011) 27:1 Computers in Human Behavior 296 [Eyrich-Garg]; David E. Pollio, D. Scott Batey, Kimberly Bender, Kristin Ferguson et Sanna Thompson, « Technology Use among Emerging Adult Homeless in Two U.S. Cities » (2013) 58:2 Soc Work 173 [Pollio et al].

118 Eyrich-Garg, ibid à la p 6; Pollio et al, ibid à la p 2.

119 Thomas H. Muggleton et Ian Ruthven, « Homelessness and access to the informational mainstream » (2012) 68:2 J Doc 218 à la p 228.

120 Pollio et al, supra note 117 à la p 2.

121 Site internet de Homeless Nation, en ligne : HomelessNation <http://www.homelessnation.org>. Voir aussi le site internet de Invisible People (aux États-Unis), en ligne : Invisible People <http://invisiblepeople.tv/blog/about/>

122 Compte Twitter de Logements Insalubres Montréal, en_ligne : Twitter <https://twitter.com/Log-eInsaMtl>.

123 Site internet de HomelessHub, en ligne : HomelessHub <http://www.homelesshub.ca/>.

124 Bouclin, supra notes 24 et 116. 


\section{LA CYBERJUSTICE COMME OUTIL D’ACCÈS À LA JUSTICE DES PERSONNES ITINÉRANTES}

Les recherches existantes sur la façon dont les médias sociaux peuvent favoriser des connections vitales pour les personnes itinérantes tendent à traiter uniquement des expériences de celles qui occupent une position (relativement) privilégiée, car elles maîtrisent déjà le numérique et partagent plusieurs caractéristiques : elles sont souvent jeunes, urbaines, blanches et de sexe masculin ${ }^{125}$. Qui plus est, les personnes qui occupent des positions (relativement) moins privilégiées dans la communauté itinérante — les femmes, les personnes âgées, les personnes ayant une déficience, celles qui ont des problèmes de santé mentale, les personnes a faible scolarité ou pour lesquelles ni le français ni l'anglais n'est une langue courante - sont moins à même de profiter des ressources disponibles en ligne. À ce sujet (mais dans le contexte de l'accès à la justice en général), la directrice générale de la Commission du droit de l'Ontario, Patricia Hughes, affirme ceci: «It appears, perhaps not surprisingly, that those for whom the technological resources are often developed — those lacking access to legal resources in other forms (most particularly, lawyers) — receive the least benefit from it. " ${ }^{126}$ Cette déclaration est d'autant plus vraie pour les personnes souffrant des désavantages et du stress additionnels formant l'apanage quotidien des personnes itinérantes, dont peu «connaissent l'état de leur situation judiciaire ou encore les différentes étapes de la procédure pénale » ${ }^{127}$.

Pour les membres de la communauté itinérante qui ont déjà accès aux médias sociaux, certains projets de cyberjustice pourraient s'avérer efficaces relativement à l'accès à la justice.

Il a été démontré que plusieurs personnes itinérantes utilisent les médias sociaux et Internet pour rester en communication avec leurs proches ${ }^{128}$. L'auteure principale a aussi démontré que les personnes itinérantes se servent de médias sociaux pour échanger de l’information sur leurs droits, pour dénoncer des injustices (comme les expulsions des espaces publics) et pour s’imposer comme critiques légitimes du système judiciaire ${ }^{129}$. Les médias sociaux permettent aussi d'engager des relations d'entraide avec un entourage ayant vécu les mêmes difficultés judiciaires ${ }^{130}$ que soi. Les médias sociaux constituent donc certainement un bon moyen de tâter le terrain et d'obtenir de l'information de base. Ils ne sont toutefois pas suffisants pour outiller complètement un individu qui souhaite s'engager dans un processus judiciaire.

Néanmoins, de telles innovations représenteraient un fardeau supplémentaire pour les organismes d'aide à l'itinérance. Nous proposons donc de construire des liens forts entre la communauté pro bono,

125 Bouclin, 2014, supra note 116; David G. Hendry et al, « How to Integrate Digital Media into a Drop-in for Homeless Young People » (2011) 33:5 Child \& Youth Serv Rev 774; Karabanow et Naylor, supra note 116.

126 Pour expliquer cette affirmation, elle émet des hypothèses liées au manque de connaissances nécessaires à l’utilisation des ressources par les personnes visées : Patricia Hughes, «Technological Means to Access to Justice » (15 septembre 2009), en ligne : Slaw <http://www.slaw.ca/2009/09/15/technological-means-to-access-to-justice/>. Raffestin, injustice programmée, supra note 20 à la p 89. Plusieurs personnes déplorent le fait d'être condamnées à la prison sans avoir rencontré de juge et affirment se sentir comme un numéro face aux procureurs de la poursuite. Une répondante a même affirmé que ces procureurs " profitent du fait que les accusés ne connaissent pas leurs droits ». Bouclin 2011, supra note 22; Eyrich-Garg, supra note 117; Muggleton, supra note 119.

129 Bouclin 2014, supra note 116.

130 Tel que l'explique Cassandra Robertson, supra note 101 à la p 84. 
les étudiants bénévoles et les organismes d'aide existants. Tous ces acteurs pourraient profiter d'un échange d'information. Par exemple, les organismes d'aide pourraient apprendre à connaître les lois et, en contrepartie, pourraient former les acteurs judiciaires sur la façon de traiter avec les clients souffrants de maladie mentale. Les propositions suivantes nécessiteront sans doute des subventions publiques, des activités de sensibilisation et de l'éducation publique à l'égard de l'itinérance. Ces constats établis, voici quelques propositions pour favoriser l'accès à la justice des personnes itinérantes.

D'abord, en collaboration avec les services pro bono, nous croyons qu'il serait possible d'utiliser Skype ${ }^{131}$ dans les cliniques mobiles qui visitent déjà les refuges d'urgence ${ }^{132}$; cette mesure permettrait à une équipe d'avocats et de parajuristes d'offrir des consultations rapides dans des cas d'expulsion imminente d'un logement, d'annulation de prestations d'aide sociale ou d'assurance-invalidité ou pour des questions liées à une contravention. Ces accompagnateurs juridiques pourraient aussi expliquer le fonctionnement des logiciels de règlement en ligne de litige (RLL) et diriger les individus vers ceux-ci lorsqu'il serait plus avantageux de régler des questions juridiques au moyen de la négociation ou de la médiation - dans le cas de l'éclatement de la cellule familiale, par exemple. Le RLL pourrait éventuellement améliorer l'agentivité ${ }^{133}$ judiciaire des personnes itinérantes en créant des forums plus accessibles pour la résolution des conflits. Cependant, il demeure difficile de savoir si ces mesures sont une solution viable au problème d'accès à la justice des personnes itinérantes puisqu'il n'existe aucune recherche sur l'utilisation par celles-ci du RLL ${ }^{134}$.

Qui plus est, certaines recherches révèlent que les logiciels de RLL posent des risques pour les personnes vulnérables ${ }^{135}$, et qu'ils ne sont pas des forums appropriés pour régler tous les types de conflits ${ }^{136}$. Dans les circonstances où les personnes itinérantes sont en contact avec des institutions légales qui, par définition, interviennent dans la vie des gens par des moyens draconiens ${ }^{137}$ (par exemple, les cas de placements et de soins non consentis en institut psychiatrique, de retrait de la garde des enfants ou de violence policière), les personnes itinérantes auront toujours avantage à recourir à une représentation juridique au sein du système judiciaire traditionnel et antagoniste qui permet l'opposition entre deux parties.

En ce qui concerne la vidéoconférence, comme cela a été noté plus haut, il s’agit d'un outil qui doit être utilisé avec doigté. Dans sa forme privilégiée actuellement (amener le processus judiciaire dans les régions éloignées en utilisant la caméra), nous ne croyons pas qu'elle facilite l'accès à la justice des personnes itinérantes. Ces personnes font déjà, de manière générale, peu confiance au système de

131 Ou un autre logiciel similaire. SKYPE est un logiciel gratuit qui permet aux utilisateurs de passer des appels téléphoniques via Internet, Site internet de Skype, en ligne : Skype <www.skype.com>.

132 Voir par ex RAPSIM, « Opération Droits Devant » (2003), en ligne : RAPSIM

<http://www.rapsim.org/129/OperationeDroitseDevant.montreal>.

133 L'étymologie du terme provient du terme latin agentia, facultas agenda - signifiant que l'agentivité est l'état ou la possibilité d’agir : The Oxford English Dictionary, 2 éd, sub verbo « agency ».

134 Il y a une crainte que les personnes vulnérables ne puissent pas participer adéquatement aux processus de RLL sans représentant, parce qu'elles ne comprennent pas suffisamment leurs droits et elles sont donc vulnérables : Larry R. Spain, « Alternative Dispute Resolution for the Poor: Is It an Alternative? » (1994) 70 NDL Rev 169. Spain, ibid; Grillo, supra note 43.

136 Spain, ibid; Grillo, ibid.

137 Aide juridique Ontario, Rapport de l'examen du Régime d'aide juridique de l'Ontario : Plan d'action pour les services juridiques publics subventionnés, 1997, en ligne :

<http://www.attorneygeneral.jus.gov.on.ca/french/about/pubs/olar/ch4.asp> au chapitre 4. 
justice. Il est possible de croire qu’à distance et à travers un écran, elles pourraient lui faire encore moins confiance $^{138}$. Cette méfiance accrue nuirait considérablement à notre objectif d'améliorer l'accès à la justice en amenant les personnes itinérantes à se familiariser avec le droit et le processus judiciaire : la vidéoconférence crée une barrière supplémentaire entre la personne et l'acteur judiciaire. Elle peut toutefois être utilisée pour permettre à des avocats de faire du bénévolat (pro bono) à distance ${ }^{139}$, augmentant du même coup l'accès aux ressources gratuites ${ }^{140}$ et privilégiant un accès à la justice. Nous croyons que la vidéoconférence pourrait s'avérer utile, dans une optique d'augmentation des ressources sociales et des conseils juridiques, aux personnes itinérantes à mobilité réduite ou résidant dans les régions éloignées ${ }^{141}$.

Pour augmenter l'accès à la cyberjustice d'autres populations itinérantes, nous pouvons par exemple penser à des locaux informatiques consacrés à la justice ${ }^{142}$. Ces locaux feraient partie d'un continuum de soutien (hébergement, logement social, soins de santé physique et mentale, garderie, etc.). Il nous semble important que les sites d'accès aux ressources informatiques et leur environnement soient adaptés de façon réaliste aux besoins et capacités de la pluralité d’individus vivant dans la rue, et que de l'aide soit facilement accessible quant à l'utilisation de ces ressources, dans un objectif d'autonomisation et d'autogestion des personnes de la rue. La situation géographique et les heures d'ouverture de ces locaux devraient être soigneusement élaborées et publicisées en partenariat avec les organismes d'aide aux personnes itinérantes ${ }^{143}$. Nous pouvons facilement envisager des centres de service de cyberjustice qui soient disponibles 24 heures sur 24 pour assister les personnes dont les horaires sont irréguliers; ces centres seraient pourvus d'un personnel composé de travailleurs sociaux, d'accompagnateurs juridiques (pour aider les bénéficiaires à remplir des formulaires ou à distinguer les questions juridiques de leurs autres besoins) et de spécialistes en informatique pouvant assister les utilisateurs des logiciels.

138 Tel que le souligne Anne Wallace dans le cas des autochtones d'Australie, supra note 52 à la p 16. Aux États-Unis, un groupe de chercheurs de l'université Harvard en est également venu à la conclusion que pour les résidents américains ne maitrisant pas la langue anglaise, il faut être très prudent avant d'instaurer des systèmes de technologies à distance, même lorsqu'on prévoit founir les services de traducteurs : James E. Cabral, Abhijeet Chavan, Thomas M. Clarke, John Greacen, Bonnie Rose Hough, Linda Rexer, Jane Ribadeneyra et Richard Zorza, « Using Technology to Enhance Access to Justice » (2012) 26:1 Harv JL \& Tech 241 à la p 264.

139 Voir Dugald E. Christie, «Blueprint for Access to Justice: A Visionary Practical Plan for B.C. and Canada » (2007) 40 UBC L Rev 455 à la p 1.

140 L'accès gratuit à un conseiller juridique pour toute personne qui en a besoin est un des objectifs à atteindre pour que l'accès à la justice soit équivalent pour tous, voir Christie, ibid à la p 2.

141 Cabral et al, supra note 138 à la p 255. Voir aussi, à la p 270, par exemple, le « Central California Legal Services » installe des ordinateurs dans plusieurs lieux éloignés du territoire californien (comme dans les centres pour personnes âgées et les centres communautaires) où les gens peuvent se rendre afin d'avoir des discussions avec des intervenants des zones urbaines.

Cela assurerait un contact humain qui, de l'avis de certains, ne pourra jamais être totalement remplacé par l'informatique, voir par ex Patricia Hughes, ibid.

143 Afin de maximiser les chances d'atteindre les personnes les plus vulnérables, voir Stanislava Gaydazhieva, « Experts discuss impact of information technology on access to justice » (7 décembre 2012), en ligne : NEWEUROPE $<$ http://www.neurope.eu/article/experts-discuss-impact-information-technology-access-justice> ( The lack of access to the Internet also negatively affected some individuals, in particularly the most vulnerable groups »). 
Dans une perspective axées sur la prévention des conflits ${ }^{144}$, l'éducation populaire et l'établissement de priorités d'intervention judiciaire ${ }^{145}$, les services juridiques offerts doivent répondre aux besoins particuliers des personnes itinérantes. L'accompagnement juridique doit tenir compte des stress et traumatismes quotidiens associés à la vie itinérante ${ }^{146}$.

Finalement, la présence sur les médias sociaux des tribunaux ${ }^{147}$, des organisations d'aide juridique ${ }^{148}$ et des organismes d'éducation juridique publique peut contribuer à améliorer l'accès à l'information juridique des personnes itinérantes. Le fait que ces entités juridiques communiquent sans l'intermédiaire d'un avocat et de façon abrégée directement avec les justiciables aide ces derniers à se familiariser avec le droit et leur offre une nouvelle façon de trouver des informations et des ressources. On se trouve ainsi à fournir aux personnes le savoir-faire nécessaire au développement d'une agentivité juridique.

\section{CONCLUSION}

Nous croyons que le développement de la cyberjustice peut contribuer à améliorer l'accès à la justice des personnes itinérantes. Il ne faut toutefois jamais perdre de vue que l'intégration des TIC ne doit pas complexifier un système qui, déjà, est difficile d'accès ; tel que le mentionne le Comité d'action sur l'accès à la justice en matière civile et familiale, «[s]i elles sont mal gérées, les réactions aux nouvelles technologies peuvent isoler les groupes et encourager la compétitivité et l'exclusivité. Si elles sont bien gérées, le partage de l'information concernant les innovations technologiques peut favoriser une collaboration bénéfique pour l'ensemble du secteur. " ${ }^{149}$ Si nous croyons donc en l'efficacité des TIC pour améliorer l'accès à la justice des personnes itinérantes, nous croyons aussi que cette efficacité ne

144 Plusieurs études ont démontré, par exemple, la judiciarisation disproportionnée des personnes en situation d'itinérance, voir par ex Commission des droits de la personne et des droits de la jeunesse, La judiciarisation des personnes itinérantes à Montréal : un profilage social par Christine Campbell et Paul Eid, en ligne : (2009) cdpdj.qc.ca $<$ http://www.cdpdj.qc.ca/publi-cations/itinerance_avis.pdf>; Secrétariat national des sans-abri, Judiciarisation et criminalisation des populations itinérantes à Montréal par Céline Bellot, Isabelle Raffestin, Marie-Noële Royer et Véronique Noël, en ligne : (2005) RAPSIM <http://www.rapsim.org/docs/rapport_Bellot_05_VF.pdf>; Bouclin, 2011, supra note 24; Suzanne Bouclin et Joëlle Pastora-Sala, « Une analyse de la règlementation des personnes itinérantes selon une perspective pothienne de la discrimination » (2013) $25: 1$ Revue femmes et droit 1.

145 C'est d'ailleurs le modèle proposé par le Homeless Legal Advocacy Project de Calgary, Site internet du Homeless Legal Advocacy Project, en ligne : Calgary Housing \& Employment Service <http://www.calgaryhes.ca/homeless-legaladvocacy-project/>.

Une vision pluraliste, humaniste et relationnelle de l'accès à la justice viserait quant à elle à améliorer l'agentivité juridique des individus et des communautés, ou la capacité de faire des choix individuels et contextualisés en fonction du vécu judiciaire d'un particulier et d'agir en conformité avec ces choix. L'auteure principale élabore présentement une théorie « humaniste » de l'accès à la justice qui engage la capacité d’une communauté à jouer un rôle actif dans la prise de décision et vise à équiper les individus et les communautés d'un savoir-faire et de ressources pour résoudre des conflits, circonscrire les responsabilités mutuelles et établir des règles générales d'interaction.

147 Voir supra notes 76, 77 et 78.

148 Voir par ex le blog d'Aide juridique Ontario, Site internet du blog d'Aide juridique’Ontario : $<$ http://blog.legalaid.on.ca/>.

149 Comité d'action sur l'accès à la justice en matières civile et familiale, L'accès à la justice en matières civile et familiale : une feuille de route pour le changement, Ottawa, Comité d'action sur l'accès à la justice en matières civile et familiale, 2013 à la p 25. 
peut être atteinte sans des consultations avec les personnes itinérantes elles-mêmes ${ }^{150}$ pour cerner leurs besoins et saisir comment y répondre adéquatement ${ }^{151}$. Cette efficacité ne peut non plus être atteinte sans l'intégration des organismes qui s'occupent de ces personnes dans une discussion sur le développement de logiciels adaptés à leurs besoins. Dans plusieurs juridictions, les organismes collaborent avec la communauté juridique pour desservir les populations itinérantes ${ }^{152}$. Nous devrions nous inspirer de modèles qui utilisent déjà la baladodiffusion ${ }^{153}$ et les médias sociaux comme Facebook $^{154}$ et Twitter $^{155}$ comme moyens de joindre les personnes itinérantes et comme outils d'éducation juridique.

Nous croyons en la pertinence du développement de centres de cyberjustice en partenariat avec les organismes concernés ${ }^{156}$, pour régler en partie les difficultés liées à la judiciarisation. Ces conclusions, que nous présentons ici comme étant des hypothèses ayant été détaillées dans la dernière section du présent article, seront testées par la chercheure principale au moyen d'un travail de terrain et d'entrevues qualitatives auprès des personnes itinérantes.

150 Cette vision d’un accès à la justice adapté aux besoins de chacun et personnalisé est la vision recommandée par Melina Buckley dans Buckley, supra note 109 à la p 14.

151 Voir par ex le programme Street Youth Legal Services, Page web du Street Youth Legal Services, en ligne : Justice for Children and Youth <http://www.jfcy.org/slys.html>.

152 Voir par ex Site internet du Chicago Coalition for the Homeless, en ligne : chicagohomeless.org <http://www.chicagohomeless.org/programs-campaigns/legal-aid/law-project/> ; Northeast Ohio Coalition for the Homeless, «Cleveland Homeless Legal Assistance Program », en ligne : Neoch.org <http://www.neoch.org/clevelandhomeless-legal-assis/>; Public Interest Advocacy Centre LTD, « Homeless Person’s Legal Service », en ligne : pias.asn.au <http://www.piac.asn.au/projects/homeless-persons-legal-service/introduction>; Bar Association of San Francisco (BASF), « Homeless Advocacy Project », en ligne : sfbar.org <http://www.sfbar.org/jdc/legalservices/hap/index.aspx>; Site internet du Shelter Legal Services, en ligne : Shelter Legal Services $<$ http://www.shelterlegalservices.org/>; Site internet du Street Law Centre Wa Inc, en ligne : Street Law Centre $<$ http://www.streetlawcentre.org.au/>.

153 Voir par ex Student Legal Services of Edmonton, « Homeless Right Street Sheets: Know your Rights », en ligne : Student Legal Services of Edmonton <http://www.slsedmonton.com/civil/homless-rights/>.

154 Voir par ex Compte Facebook du Homeless Persons Legal Service, en ligne : Facebook $<$ https://www.facebook.com/pages/Homeless-Persons-Legal-Service/144854328875834>.

155 Voir par ex Compte Twitter de Homeless Law, en ligne : Twitter <https://twitter.com/homelesslaw $>$.

156 Un tel partenariat s'inscrit dans l'optique de la recommandation faite par le Comité d'action sur l'accès à la justice en matières civile et familiale (CMOAJ), supra note 149 à la p 23. 